\title{
Characterization of Mode I Fracture and Morphological Properties of PLLA Blends with Addition of Lysine Triisocyanate*
}

\author{
Vilay VANNALADSAYSY** and Mitsugu TODO*** \\ ** Department of Mechanical Engineering, Faculty of Engineering, National University of Laos, \\ P.O.Box 3166, Vientiane, Laos \\ E-mail: villayv@gmail.com \\ *** Research Institute for Applied Mechanics, Kyushu University, \\ 6-1 Kasuga-koen, Kasuga, 816-8580, Japan
}

\begin{abstract}
Poly(L-lactic acid) (PLLA) was toughened by blending with three different ductile biopolymers such as poly ( $\varepsilon$-caprolactone) (PCL), poly(butylene succinate-co-e-caprolactone) (PBSC), poly (butylene succinate-co-L-lactate) (PBSL). The blend ratio was fixed to 50:50. Lysine triisocyanate (LTI) was added to the blends as a compatibilizer. Characterizations such as Fourier transform infra-red (FT-IR) spectroscopy, field-emission electron microscope (FE-SEM), and mode I fracture test were used to characterize the effectiveness of LTI on the mechanical and morphological properties of various PLLA blends. It was found that PLLA/PCL blend shows the highest toughness energy among the binary blends. On the other hand, addition of LTI in PLLA/PBSC blend exhibits the best toughness property. Based on the FE-SEM observation, fractured surfaces of PLLA blends with LTI indicate ductile fracture with dense elongated fibrils. The largest damage zone is generated in the vicinity of crack-trip, suggesting that high energy dissipation occurred in the crack-trip region. FT-IR analysis also suggested that the NCO groups of LTI were acted as a compatibilizer, as the results of interaction between the two phases of the polymer blends.
\end{abstract}

Key words: Biodegradable Plastics, Blend, Poly (L-Lactic Acid), Poly

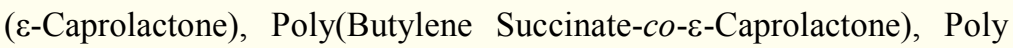
(Butylene Succinate-co-L-Lactate), Phase-Separation

\section{Introduction}

Biodegradable polyesters such as poly(L-lactic acid) (PLLA) has been attracting much attention because it is biodegradable, compostable, producible from renewable resources, and nontoxic to the environment and the human body [1-15]. PLLA has also been used for automobiles, electric appliances, food packages and medical devices. Fracture properties and behavior of PLLA have been investigated, and it was found that PLLA exhibits relatively brittle fracture behavior in nature [5-7]. In order to improve such properties, the blends of PLLA with other flexible and biodegradable polyesters such as PBS [8], PBSL [9], PBSC [10] and PCL $[5,6,8]$ have been investigated. The PLLA-based blends with those ductile biodegradable polymers have been developed to improve the fracture properties of neat PLLA, and it was found that the blends exhibit better properties than neat PLLA [5-10]. It was also found that those PLLA blends create phase-separation morphology caused by immiscibility of the components. As an effective method to improve the phase-separation of the PLLA blends, we have introduced compatibilizers such as lysine triisocyanate (LTI) and 
PEO-PPO-PEO copolymer $[10,14,15]$. We have found that the addition of LTI or PEO-PPO-PEO to PLLA/PBSL improved the fracture toughness of the blends, and LTI is more effective than PEO-PPO-PEO copolymer. Based on the previous studies, the blend ratio of 50/50 in weight ratio with addition of LTI 2 phr was chosen to characterize and compare the mode I fracture properties and morphology of three different kinds of biodegradable polymer blends, namely, PLLA/PCL, PLLA/PBSL and PLLA/PBSC. Suitability of three ductile polymers as a blending partner for PLLA was also assessed based on the experimental results. Mode I fracture mechanisms were also investigated by observing the fracture surfaces using a field emission scanning electron microscope (FE-SEM). FT-IR analysis was also performed to characterize the interaction between the two phases of the polymer blends.

\section{Experimental}

\subsection{Materials and specimens}

PLLA pellets $\left(\right.$ Lacty $^{\circledR} \# 5000 ; \mathrm{M}_{\mathrm{w}}=1.45 \times 10^{5}, T_{\mathrm{g}}=60^{\circ} \mathrm{C}, T_{\mathrm{m}}=180{ }^{\circ} \mathrm{C}$ ) were supplied by Toyota Motor Co., Ltd. PCL pellets (Celgreen PH7; $M_{\mathrm{w}}=1.25 \times 10^{5}, T_{\mathrm{g}}=-60^{\circ} \mathrm{C}$ and $T_{\mathrm{m}}=61$ $\left.{ }^{\circ} \mathrm{C}\right)$ were supplied by Daicel Chemical Industries Ltd. PBSC pellets $\left(M_{\mathrm{w}}=1.7 \times 10^{5}, T_{\mathrm{g}}=-42\right.$ ${ }^{\circ} \mathrm{C}, T_{\mathrm{m}}=101{ }^{\circ} \mathrm{C}$ ) were supplied by Daicel Chemical Industries, Ltd. PBSL pellets (GS Pla ${ }^{\circledR}$ AZ-type; $M_{\mathrm{w}}=1.47 \times 10^{5}, T_{\mathrm{g}}=-33{ }^{\circ} \mathrm{C}, T_{\mathrm{m}}=112{ }^{\circ} \mathrm{C}$ ) were obtained from Mitsubishi Chemical Co., Ltd. These pellets were held into a desiccator to keep them dry and prevent from degradation due to hydrolysis by moisture. High viscous solution of LTI was supplied by Kyowa Co., Ltd.

Blend ratio of PLLA and PCL (or PBSC, PBSL) was fixed at 50/50 wt $\%$ with and without addition of LTI (2phr). The blends were prepared by melt-mixing in a conventional melt-mixer at $190{ }^{\circ} \mathrm{C}$ and a rotor speed of $50 \mathrm{rpm}$ for $20 \mathrm{~min}$. The mixtures were then compression molded at $30 \mathrm{MPa}$ at $190{ }^{\circ} \mathrm{C}$, and then followed by cooling process using a

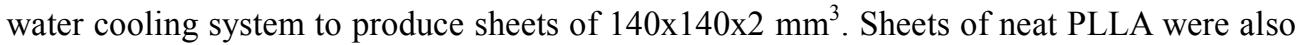
fabricated through the same molding process. Single-edge-notch-bend (SENB) specimens for mode I fracture test was then prepared from these sheets. The dimension of testing samples was approximately $50 \mathrm{~mm} \times 10 \mathrm{~mm} \times 2 \mathrm{~mm}$. Table 1 exhibits the sample labeling with corresponding compositions of the blends.

Table 1 Sample Designation and Composition of PLLA Blends

\begin{tabular}{llll}
\hline Sample Code & Composition & Weight Percent $(\mathbf{w t} \%)$ & LTI (phr) \\
\hline PLLA & PLLA & 100 & 0 \\
PCL & PCL & 100 & 0 \\
PBSL & PBSL & 100 & 0 \\
PBSC & PBSC & 100 & 0 \\
AL 50 & PLLA/PCL & $50 / 50$ & 0 \\
ALI 50 & PLLA/PCL & $50 / 50$ & 2 \\
ASL 50 & PLLA/PBSL & $50 / 50$ & 0 \\
ASLI 50 & PLLA/PBSL & $50 / 50$ & 2 \\
ASC 50 & PLLA/PBSC & $50 / 50$ & 0 \\
ASCI 50 & PLLA/PBSC & $50 / 50$ & 2 \\
\hline
\end{tabular}

\subsection{Analysis methods}

The Fourier Transform Infra-red (FT-IR) measurement was carried out using Perkin Elmer Spectrum One spectrometer with resolution of $2 \mathrm{~cm}^{-1}$ for 4 scans over wave number range of $400-4000 \mathrm{~cm}^{-1}$. The film samples were obtained by casting polymer resolution on 
the KRS-5 disk.

Mode I fracture tests of the SENB specimens were performed at a loading rate of 1 $\mathrm{mm} / \mathrm{min}$ by using the servo-hydraulic testing machine. The histories of the load and the displacement at the loading point were recorded using a digital recorder. The resulting load-displacement relationships were obtained. Mode I fracture properties, such as the initial fracture energy, $J_{i n}$, which is defined as the critical $J$-integral value at crack initiation, and the averaged fracture energy, $J_{f}$, were then evaluated using the following Equations [15]:

$$
\begin{aligned}
& J_{i n}=\frac{\eta U_{\text {in }}}{B(W-a)} \\
& J_{f}=\frac{U_{f}}{B(W-a)}
\end{aligned}
$$

where $U_{\text {in }}$ is the critical energy at crack initiation, which is defined as the point where the stiffness of the specimen starts to rapidly decrease; $U_{\mathrm{f}}$ is the total fracture energy that is dissipated by the complete fracture of the specimen; $B$ and $W$ are the specimen thickness and width, respectively; $a$ is the initial crack length; and $\eta$ is the geometrical correction factor and $\eta=2$ for the standard SENB specimen. At least five specimens were tested to obtain the average and standard deviation (SD).

The microstructures of the blends were investigated by using a high-resolution field emission scanning electron microscope (FE-SEM). Cryo-fracture surfaces were obtained by immersing the specimens in liquid nitrogen for about 30 minutes and then observed by FE-SEM. Fracture surfaces of SENB specimens were also observed by FE-SEM to characterize fracture mechanism and effect of LTI addition on fracture behavior.

\section{Results and Discussion}

\subsection{FT-IR Analysis}

Figure 1 shows the FT-IR spectra of neat PLLA, PCL, PBSL, PBSC and the PLLA blends. The characteristic peak of the hydroxyl group (-OH) appears in the region $3440-3500 \mathrm{~cm}^{-1}$. As the end-group, their intensities consistently appeared quite weakly due to their low molar content in the respective polymeric chains. Two distinct peaks in the regions $2858-2995 \mathrm{~cm}^{-1}$ correspond to the asymmetrical and symmetrical stretching mode of the $\mathrm{CH}_{3}$ groups. In all spectra, the $\mathrm{C}=\mathrm{O}$ group occurred in the region $1751-1758 \mathrm{~cm}^{-1}$, while that of $\mathrm{C}-\mathrm{O}-\mathrm{C}$ occurred in the region $1050-1200 \mathrm{~cm}^{-1}$. Peaks in the region $1430-1480 \mathrm{~cm}^{-1}$ represent $\mathrm{C}-\mathrm{H}$ deformation, while those at $733-756 \mathrm{~cm}^{-1}$ represent the skeletal vibration of the methylene groups.

Based on the FT-IR analysis of these blends, the peak in the region $1720-1760 \mathrm{~cm}^{-1}$ which corresponds to $\mathrm{C}=\mathrm{O}$ group appeared in doublet with high intensity. This doublet represent that of PLLA and PCL (PBSL and PBSC), respectively. For AL 50, double peaks are observed at $1757 \mathrm{~cm}^{-1}$ (PLLA) and $1726 \mathrm{~cm}^{-1}$ (PCL) (Fig. 1-b). For ASL 50, the peaks are observed at $1756 \mathrm{~cm}^{-1}$ (PLLA) and $1715 \mathrm{~cm}^{-1}$ (PBSL) (Fig. 1-d), while peaks of ASC 50 are observed at $1757 \mathrm{~cm}^{-1}$ (PLLA) and $1715 \mathrm{~cm}^{-1}$ (PBSC) (Fig. 1-f). This refers to motion arising from two different carbonyl groups from respective components of the binary blends between PLLA and ductile polymers of PCL, (PBSL and PBSC), respectively. Such double peaks indicate incompatible blend which display immiscibility [11].

However, after addition of 2phr LTI, these peaks shift to single broad peaks (Fig. 2), which indicate an identical bond motion in carbonyl group between the two copolymers. This can be attributed to the effect of the LTI, which formed good polar interaction between both PLLA and PCL (PBSL and PBSC). This interaction induces the mobilities of the 
respective carbonyl bonds as unit entity. This is in agreement with the previous works which showed changes in peak shift and intensity as the results of interchange of interaction due to the secondary process [12].

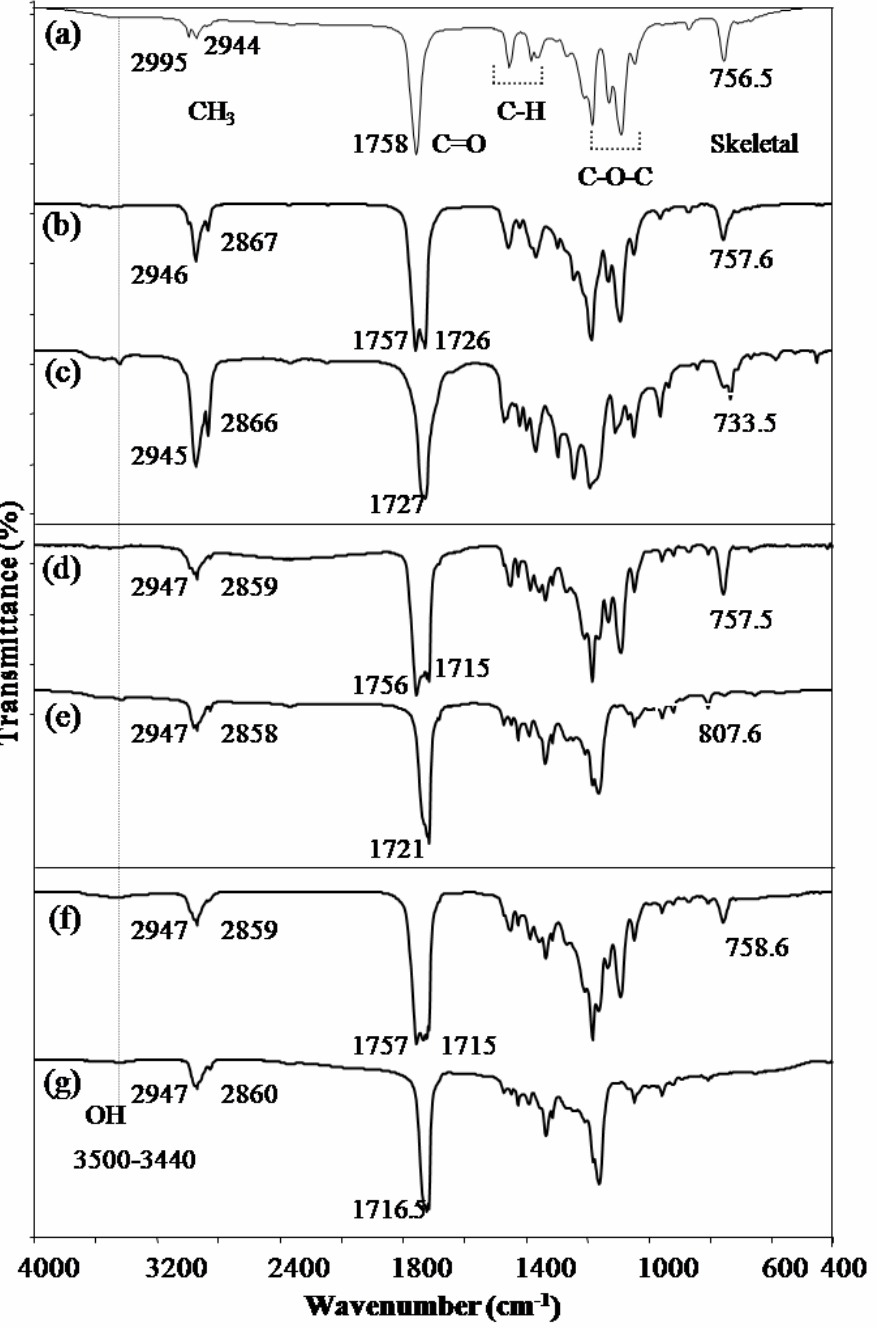

Fig. 1 FT-IR spectra of (a) PLLA, (b) AL 50, (c) PCL, (d) ASL 50, (e) PBSL, (f) ASC 50 and (g) PBSC

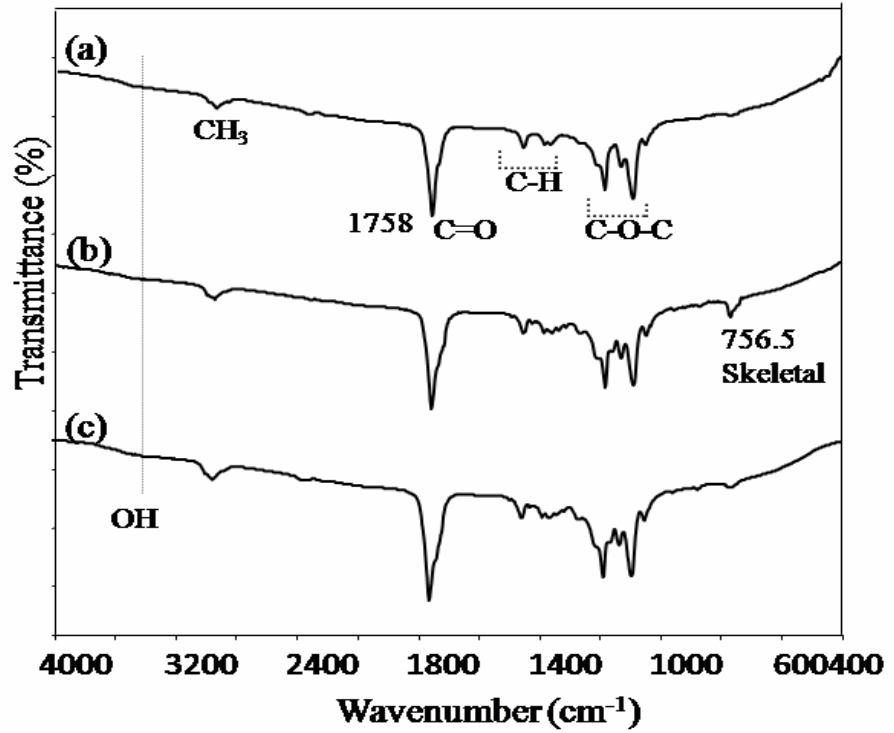

Fig. 2 FT-IR spectra of (a) ALI 50, (b) ASLI 50 and (c) ASCI 50 


\subsection{Morphological studies}

FE-SEM micrographs of the cryo-fractured surfaces are shown in Fig. 3. All the blends without addition of LTI exhibit phase separation with evolution of spherical morphology. The large spherical structures constructed the secondary phase and therefore corresponded to PCL, PBSL and PBSC spherical. This kind of morphological result agrees with immiscible polymer blends that generally create macro-phase separation of the two components due to difference of solubility parameter [13]. Such phase separation usually affects the physical and mechanical properties of the blend [11]. On the other hand, PLLA/PCL shows the smallest size of spherical PCL domains. However, PLLA/PBSL and PLLA/PBSC exhibit interfacial debonding in the surroundings of the PBSL and PBSC domains. It is thus presumed that PCL has the better miscibility with PLLA than the other polymers. On the contrary, for the blends with addition of LTI, apparent spherical structures of respective polymers could not be observed on the surfaces. This is due to improvement of miscibility between PLLA and PCL (PBSC and PBSL). Such microstructural improvement is understood to be caused by the polar interaction and hydrogen bond as discussed based on the FT-IR results.

(a)

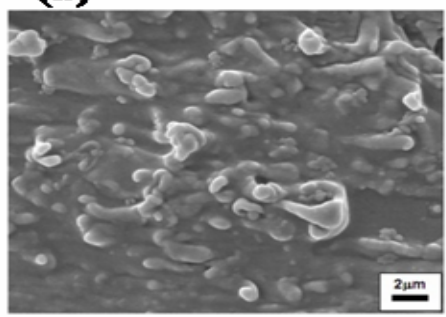

AL 50

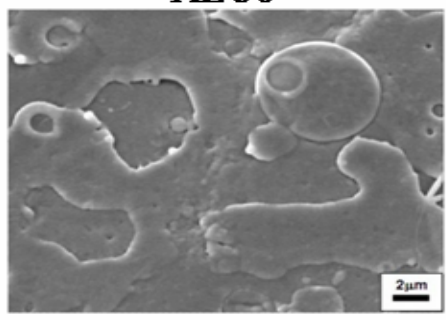

ASL 50

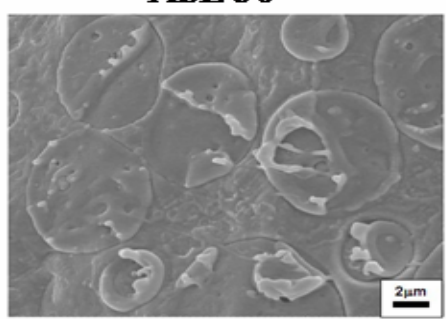

$\operatorname{Asc} 50$

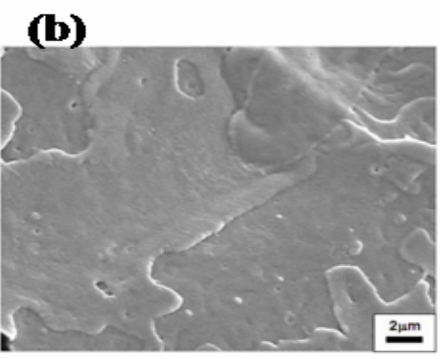

ALI 50

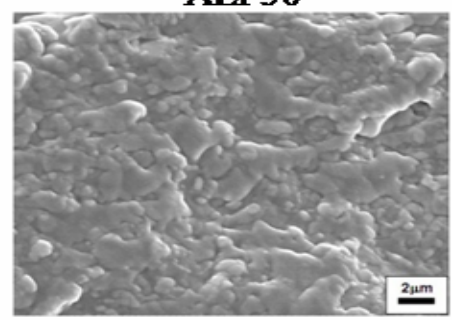

ASLI 50

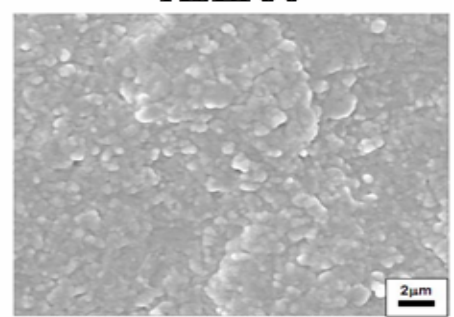

ASCI 50

Fig. 3 FE-SEM micrographs fractured surfaces under liquid nitrogen of (a) binary blends and (b) trinary blends

\subsection{Mode I Fracture Property}

Fig. 4 exhibits the load-displacement curves obtained from the Mode I fracture testing. It is clearly seen that the addition of LTI to the blends results in improvement of the mechanical behavior compared to the original blends and neat PLLA. The sudden drop at the maximum load for PLLA corresponds to a brittle fracture behavior. The decreasing behavior of load of PLLA/PBSL and PLLA/PBSC are similar with that of neat PLLA, while PLLA/PCL exhibits much more gentle slope of decreasing load corresponding to 
ductile fracture behavior. No significant difference is observed in the curve patterns of the three blend systems with LTI. However, PLLA/PBSC/LTI displays the largest maximum load and PLLA/PCL/LTI shows the smallest maximum load in the three blend systems with LTI.

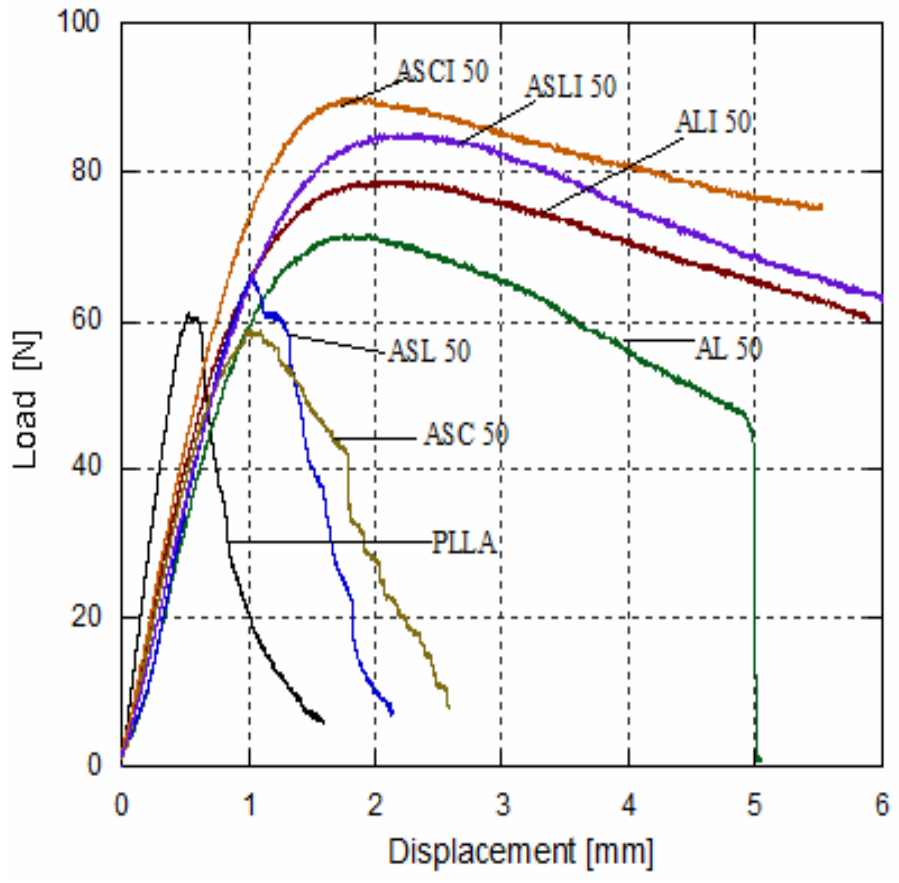

Fig. 4 Typical load-Displacement curves.

The effects of LTI addition on the initial fracture energy, $J_{\text {in }}$, and the averaged fractured energy, $J_{\mathrm{f}}$, are shown in Fig. 5. It is seen that $J_{\text {in }}$ and $J_{\mathrm{f}}$ values of PLLA/PBSL and PLLA/PBSC are slightly higher than that of neat PLLA, however, PLLA/PCL shows much higher $J_{\text {in }}$ and $J_{\mathrm{f}}$ than PLLA/PBSL and PLLA/PBSC, indicating that PLLA has better miscibility with PCL than PBSL and PBSC as consistent with cryo-fracture morphology results. In contrast, the $J_{\text {in }}$ and $J_{\mathrm{f}}$ values of the blends with LTI are obviously higher than that of the original blends and neat PLLA. PLLA/PBSC/LTI shows the largest $J_{\text {in }}$ and $J_{\mathrm{f}}$ values and followed by PLLA/PBSL/LTI and PLLA/PCL/LTI. These experimental results clearly exhibit that such LTI modification effectively improves the mode I fracture properties of PLLA polymer blends. The improvement of $J_{\mathrm{f}}$ is more significant than that of $J_{\text {in }}$, corresponding to the drastic change of crack growth behavior from brittle to ductile manner as shown in Fig. 4.

The FE-SEM micrographs of the mode I fracture surfaces in the crack propagation regions are shown in Fig. 6. It is apparent that the fracture surface of PLLA is flat and smooth corresponding to a low dissipated energy during crack propagation as shown in Fig. 6-a. On the other hand, the binary blends exhibit much rougher surfaces than PLLA and two-phase morphology is obviously constructed by the existence of many voids and fibril structures as shown in Fig. 6-b. It is naturally understood that these voids are created by removal of PCL (PBSL and PBSC) spherulites. It is also noted that the voids are created due to debonding at the interfaces between the spherulites and the matrix under a low stress level because of the lower interfacial strength than the strength of the base polymer. It is easily understood that these voids cause localized stress concentration in the surrounding matrix and therefore, accelerates both fracture initiation and propagation in the notch-tip regions. However, when LTI is added to the mixture of PLLA and PCL (PBSL and PBSC), the fracture surfaces turn to dense fibril structures (Fig. 6-c), indicating that the 
immiscibility of the polymers is greatly improved and the fracture energy dramatically increases due to entangled molecular structures.

\section{Conclusion}

Effects of LTI addition on the fracture property and the microstructural morphology of polymer blends of PLLA and PCL, PBSL and PBSC were investigated. Significant improvement in the mode I fracture energy can be achieved by LTI addition. On the fracture surfaces of the blends without LTI, many voids are observed, on the contrary, such void formation is suppressed by LTI addition due to increase of entanglement of PLLA andrespective polymer molecules caused by chemical reaction. This kind of morphological change due to LTI addition results in the dramatic improvement of the fractured energies. For the blends with LTI, spherical structures of PCL (PBSL and PBSC) could not be
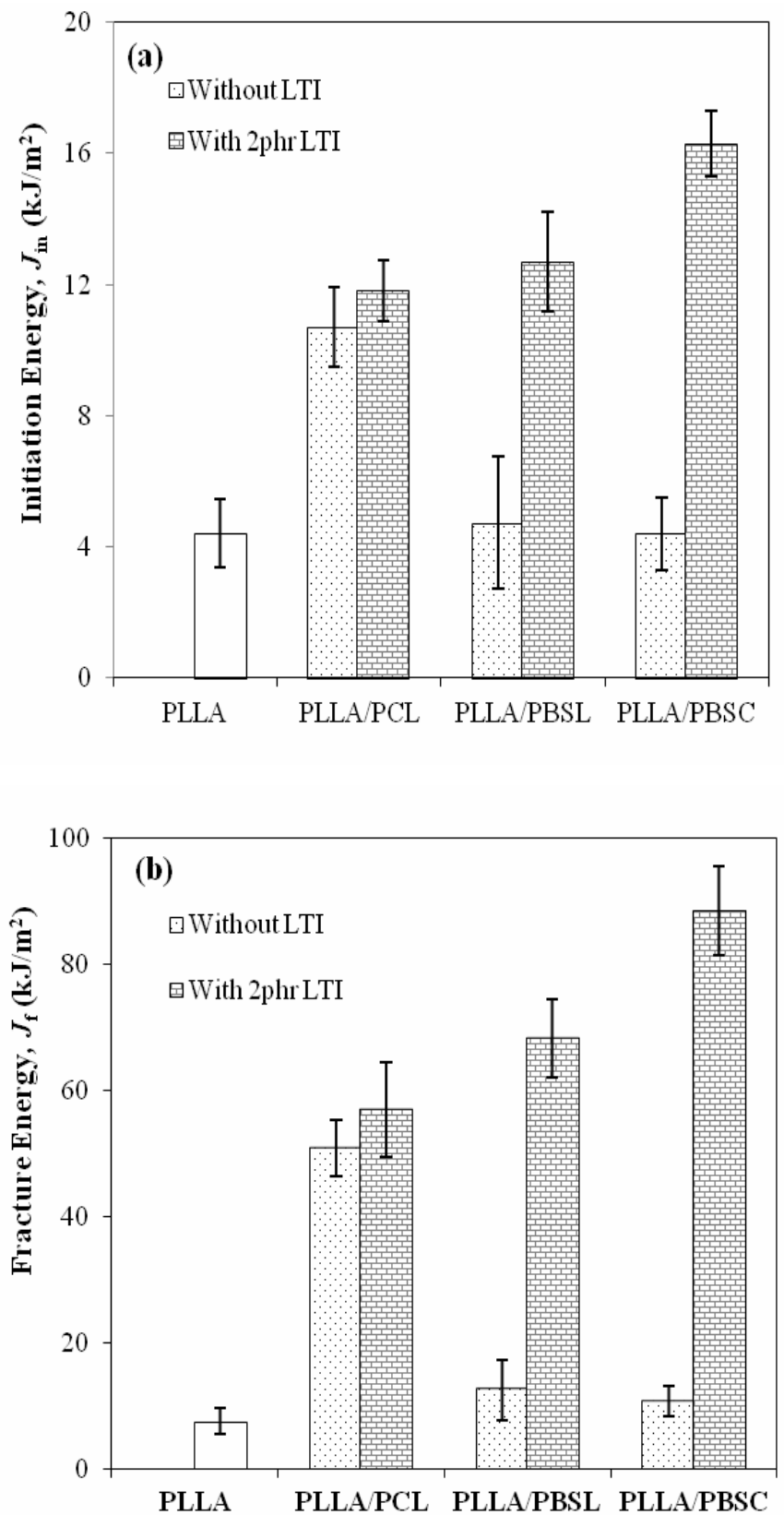

Fig. 5 Effect of LTI addition on the (a) initiation energy and (b) fracture energy values. 


\section{(a)}

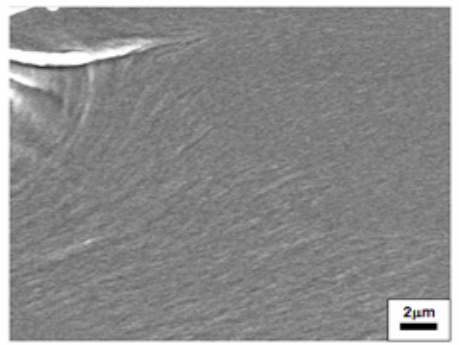

(b)

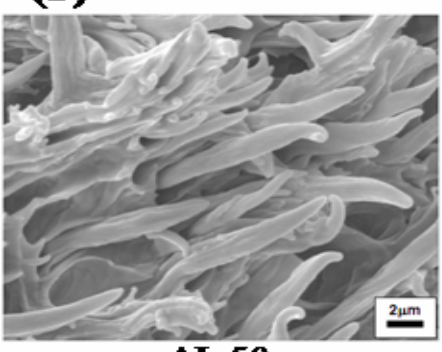

AL 50

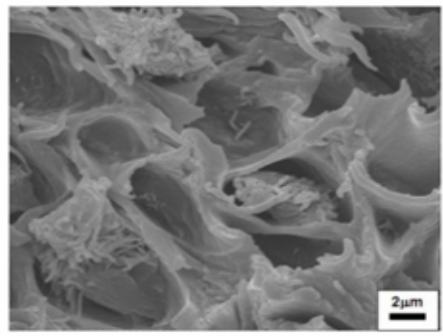

ASL 50

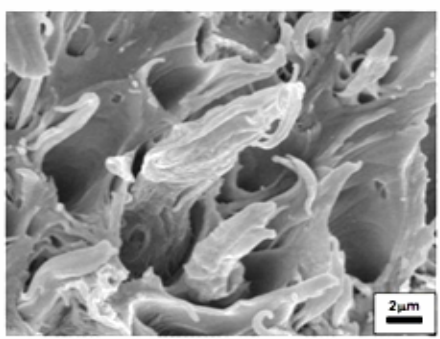

$\operatorname{Asc} 50$ (c)

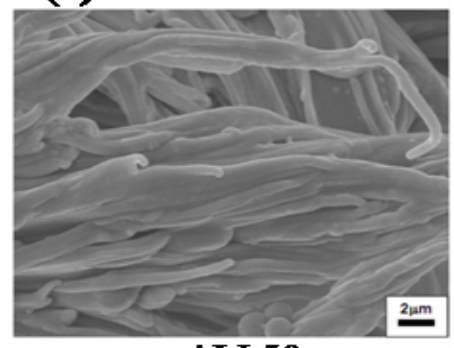

ALI 50

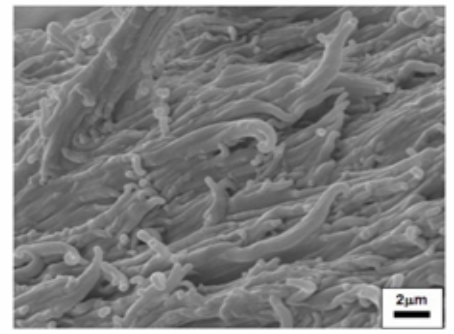

ASLI 50

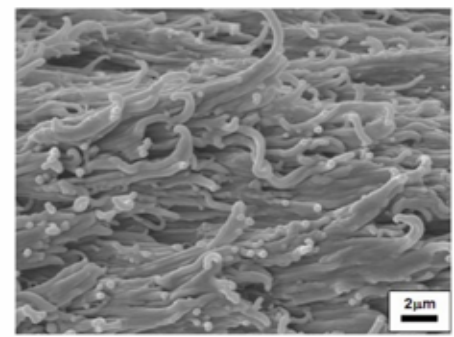

ASCI 50

Fig. 6 FE-SEM micrographs of mode I fractured surfaces of (a) neat PLLA, (b) binary blends and (c) trinary blends.

observed on the cryo-fractured surfaces by FE-SEM. This corresponds to the improvement of immiscibility between PLLA and PCL (PBSL and PBSC) due to LTI addition. FT-IR results suggest that the NCO groups of LTI are chemically reacted with the hydroxyl groups of the two polymers PLLA and PCL (or PBSL, PBSC). PLLA/PBSC/LTI blend shows the highest initiation and fracture energies, suggesting that LTI works as a compatibilizer connecting between PLLA and PBSC molecules better than PCL and PBSL.

\section{References}

(1) Garlotta, D., A Literature Review of Poly(Lactic Acid), Journal of Polymer Environment, Vol. 9, No. 2 (2001), pp. 63-84.

(2) Fujimaki, F., Processability and properties of aliphatic polyesters, 'BIONOLLE', 
synthesized by polycondensation reaction, Polymer Degradation and Stability, Vol. 59 No. 1 (1998), pp. 209-214.

(3) Goldberg, D., A review of the biodegradability and utility of poly(caprolactone), Journal of Environment Polymer Degradation, Vol. 3, No. 2 (1995), pp. 61-67.

(4) Shibata, M., Inoue, Y. and Mi Yoshi, M., Mechanical properties, morphology, and crystallization behavior of blends of poly(L-lactide) with poly(butylene succinate-co-L-lactate) and poly(butylene succinate), Polymer, Vol. 7, No 10 (2006), pp. 3557-3564.

(5) Takayama, T. and Todo, M., Improvement of impact fracture properties of PLA/PCL polymer blend due to LTI addition, Journal of Materials Science, Vol. 41, No. 15 (2006), pp. 4989-4992.

(6) Takayama, T. and Todo, M., Tsuji H. and Arakawa, K., Effect of LTI content on impact fracture property of PLA/PCL/LTI polymer blends, Journal of Materials Science, Vol. 41, No. 19 (2006), pp. 6501-6504.

(7) Todo, M., Shinohara, N. and Arakawa, K., Effects of Crystallization and Loading-rate on the Mode I Fracture Toughness of Biodegradable Poly(lactic acid), Journal of Materials Science Letters, Vol. 21, No. 15 (2002), pp.1203-1206.

(8) Park, J.W., Im, S.S., Phase behavior and morphology in blends of poly(L-lactic acid) and poly(butylene succinate), Journal Applied Polymer Science, Vol. 86, No. 3 (2002), pp. 647-655.

(9) Vilay, V., Mariatti, M., Ahmad, Z., Pasomsouk, K., Todo, M., Characterization of the Mechanical and Thermal Properties and Morphological Behavior of Biodegradable Poly(L-lactide)/Poly(e-caprolactone) and Poly(L-lactide)/Poly(butylene succinate-co-L-lactate) Polymeric Blends, Journal Applied Polymer Science, Vol. 114, No. 3 (2009), pp. 1784-1792.

(10) Vannaladsaysy, V., Todo, M., Takayama, T., Mariatti, M., Ahmad, Z., Pasomsouk, K., Effects of lysine triisocyanate on the mode I fracture behaviour of polymer blend of poly (L-lactic acid) and poly (butylene succinate-co-L-lactate), Journal of materials Science Letters, Vol. 44, No. 11 (2009), pp. 3006-3009.

(11) Dell'Erba, Ramiro., Gabriel, Groeninckx., Giovanni, Maglio., Mario, Malinconico., Anna Migliozzi., Immiscible polymer blends of semicrystalline biocompatible components: thermal properties and morphology analysis of PLLA/PCL blends, Polymer. Vol. 42, No. 18 (2001), pp. 7831-7840.

(12) Dong, J. and Ozaki, Y., FTIR and FT-Raman Studies of Partially Miscible Poly(methyl methacrylate)/Poly(4-vinylphenol) Blends in Solid States, Macromolecules, Vol. 30, No. 2 (1997), pp. 286-292.

(13) Todo, M., Park, S.D., Takayama, T. and Arakawa, K., Fracture micromechanisms of bioabsorbable PLLA/PCL polymer blends, Engineering Fracture Mechanics, Vol. 74, No. 12 (2007), pp. 1872-1883.

(14) Vilay, V., Mariatti, M., Ahmad, Z., Pasomsouk, K., Todo, M., Effect of PEO-PPO-PEO copolymer on the mechanical and thermal properties and morphological behavior of biodegradable poly(L-lactic acid) (PLLA) and poly butylene succinate-co-L-lactate) (PBSL) blends, Polymers Advanced and technologies, 2010. (http://onlinelibrary.wiley.com/doi/10.1002/pat.1672/pdf)

(15) Todo, M., Takayama, T., and Tsuji, H, Effect of LTI blending on fracture properties of PLA/PCL polymer blend, Journal of Solid Mechanics and Materials Engineering, Vol.1, No.9 (2007), pp.1157-1164. 